\title{
Obstructive sleep apnea and the prevalence and incidence of cancer
}

\author{
Tetyana Kendzerska MD PhD, Richard S. Leung MD PhD, Gillian Hawker MD MSc, George Tomlinson PhD, \\ Andrea S. Gershon MD MSc
}

\begin{abstract}
- ABSTRACT
Background: A link between obstructive sleep apnea and cancer development or progression has been suggested, possibly through chronic hypoxemia, but supporting evidence is limited. We examined the association between the severity of obstructive sleep apnea and prevalent and incident cancer, controlling for known risk factors for cancer development.

Methods: We included all adults referred with possible obstructive sleep apnea who underwent a first diagnostic sleep study at a single large academic hospital between 1994 and 2010. We linked patient data with data from Ontario health administrative databases from 1991 to 2013. Cancer diagnosis was derived from the Ontario Cancer Registry. We assessed the cross-sectional association between obstructive sleep apnea and prevalent cancer at the time of the sleep study (baseline) using logistic regression analysis. Cox regression models were used to investigate the association between obstructive sleep apnea and incident cancer among patients free of cancer at baseline.
\end{abstract}

Results: Of 10149 patients who underwent a sleep study, $520(5.1 \%)$ had a cancer diagnosis at baseline. Over a median follow-up of 7.8 years, $627(6.5 \%)$ of the 9629 patients who were free of cancer at baseline had incident cancer. In multivariable regression models, the severity of sleep apnea was not significantly associated with either prevalent or incident cancer after adjustment for age, sex, body mass index and smoking status at baseline (apnea-hypopnea index > 30 v. < 5: adjusted odds ratio [OR] 0.96, $95 \%$ confidence interval [Cl] $0.71-1.30$, for prevalent cancer, and adjusted hazard ratio [HR] $1.02,95 \% \mathrm{Cl} 0.80-1.31$, for incident cancer; sleep time spent with oxygen saturation $<90 \%$, per 10-minute increase: adjusted OR 1.01, 95\% Cl 1.00-1.03, for prevalent cancer, and adjusted HR $1.00,95 \% \mathrm{Cl} 0.99-1.02$, for incident cancer).

Interpretation: In a large cohort, the severity of obstructive sleep apnea was not independently associated with either prevalent or incident cancer. Additional studies are needed to elucidate whether there is an independent association with specific types of cancer.
$\mathrm{O}$ bstructive sleep apnea is a sleep-related breathing disorder characterized by repetitive episodes of upper-airway obstruction during sleep. Through sleep fragmentation, hypoxemia, hypercapnia, swings in intrathoracic pressure and increased sympathetic activity, these episodes lead to symptoms and health consequences. ${ }^{1}$ In 2009, $23 \%$ of Canadian adults reported risk factors for obstructive sleep apnea, and 5\% of the population 45 years and older reported being told by a health professional that they had the condition. ${ }^{2}$

Obstructive sleep apnea has been postulated to cause cancer ${ }^{3,4}$ or cancer progression, ${ }^{5}$ possibly through chronic intermittent hypoxemia, ${ }^{6}$ thus making it a potential modifiable risk factor for cancer development. ${ }^{7}$ However, the longitudinal evidence on this association is limited. Four cohort studies evaluated the longitudinal association between obstructive sleep apnea (expressed by the apnea-hypopnea index, oxygen desaturation or symptoms) and cancer development or cancer-related mortality (Appendix 1, available at www.cmaj.ca/lookup/suppl/doi :10.1503/cmaj.140238/-/DC1). ${ }^{3-5,8}$ All had limitations. Of the 3 that reported a positive association, ${ }^{3,5,8} 2$ studies included a small number of participants with severe obstructive sleep apnea, had a relatively small number of events and did not consider competing risk of death from other causes; ${ }^{5,8}$ and 2 used less reliable sleep-testing devices to define obstructive sleep apnea, ${ }^{3,8}$ which may have introduced measurement bias. In the only study that did not show an association between obstructive sleep apnea and cancer, ${ }^{4}$ the former was diagnosed on the basis of self-reported symptoms, which could have resulted in misclassification of exposure.

There is a need for a sufficiently large cohort study with a long enough follow-up to allow for
Competing interests: None declared.

This article has been peer reviewed.

Correspondence to: Tetyana Kendzerska, tetyana.kendzerska@mail .utoronto.ca

CMAJ 2014. DOI:10.1503 /cmaj.140238 
the potential development of cancer that adjusts for important potential confounders, examines common cancer subtypes and has a rigorous assessment of both obstructive sleep apnea and cancer. ${ }^{7,9,10}$ Our study was designed to improve upon the methods of published studies. We examined the association between the severity of obstructive sleep apnea (expressed by the apneahypopnea index or oxygen desaturation) and prevalent or incident cancer after controlling for known cancer risk factors.

\section{Methods}

\section{Study design and patient population}

We conducted a historical cohort study using linked clinical data from St. Michael's Hospital, Toronto, collected between 1994 and 2010 and Ontario health administrative data from 1991 to March, 2013. Ethics committees from all institutions involved approved the study (St. Michael's Hospital, Sunnybrook Research Institute, University of Toronto).

We included all patients who underwent a first diagnostic sleep study during the defined study period and who had a diagnosis of obstructive sleep apnea (apnea-hypopnea index $\geq 5$ ) or suspected obstructive sleep apnea (referred with sleep apnea, but apnea-hypopnea index $<5$ ). Details about this cohort are reported elsewhere. ${ }^{11}$

\section{Data sources}

The Sleep Laboratory database at St. Michael's Hospital includes a large set of clinical, demographic and polysomnographic variables that have been collected consistently for research purposes since 1991 (further details are available in Appendix 2, at www.cmaj.ca/lookup/suppl/ doi:10.1503/cmaj.140238/-/DC1). Each patient in the cohort underwent full in-laboratory polysomnographic recording. Standardized questionnaires were used to collect disease-specific symptoms, and a physical examination was performed by a sleep technician.
Ontario has a universal health care system that covers all medically necessary services; patient-level information is collected for administrative purposes and is available in a few large databases housed at the Institute for Clinical Evaluative Sciences. Cancer diagnoses were identified using the Ontario Cancer Registry, which contains information on all Ontario residents in whom cancer has been newly diagnosed, except non-melanoma skin cancer. ${ }^{12}$ Data in the Ontario Cancer Registry are from multiple sources and are combined to provide incidence data of good quality and completeness. ${ }^{12-15}$ Descriptions of the other datasets used in the study are available in Appendix 2.

\section{Predictive variables}

To test the hypothesis that severity of obstructive sleep apnea was associated with cancer development, we considered the apnea-hypopnea index and sleep time spent with an oxygen saturation of less than $90 \%$ as key predictors.

The apnea-hypopnea index was defined as the number of apnea and hypopnea episodes per hour of sleep. Hypopnea was consistently defined during the study period as (a) a decrease of more than $50 \%$ in the baseline amplitude of breathing for at least 10 seconds; or (b) a clear but smaller decrease in amplitude for at least 10 seconds that is associated with either an oxygen saturation drop of at least $3 \%$ or an arousal. ${ }^{16}$ The severity of obstructive sleep apnea was classified as mild (apnea-hypopnea index 5-14.9), moderate (apnea-hypopnea index 15-30) or severe (apnea-hypopnea index > 30). ${ }^{17}$

\section{Outcome measures}

We examined 2 outcomes: prevalent cancer at the time of the diagnostic sleep study (baseline) in the full cohort; and, among patients free of cancer at baseline, time from sleep study to diagnosis of incident cancer (Figure 1). The date of cancer diagnosis was the date of first diagnosis of a primary cancer, as derived from the Ontario

\section{Prevalence study}

Sample: full cohort

Design: cross-sectional

Outcome: being diagnosed with cancer 1

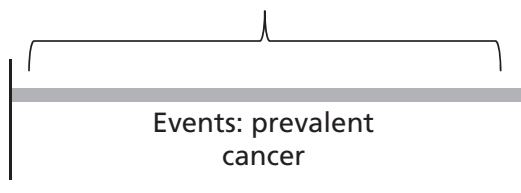

Index date: diagnostic sleep study (1994-2010)
Incidence study

Sample: cohort free of cancer at baseline Design: longitudinal

Outcome: time from sleep study to incident cancer

1

\begin{tabular}{c|}
$\begin{array}{c}\text { Events: incident cancer, } \\
\text { all-cause death }\end{array}$ \\
\hline
\end{tabular}

March 2011

Figure 1: Study design used to evaluate the association between severity of obstructive sleep apnea and prevalence and incidence of cancer. 
Cancer Registry. Patients were censored if they did not have an event of interest by the end of March 2013 or died from any cause.

\section{Potential confounders and risk factors}

We obtained data on the following known risk factors for cancer development ${ }^{18-22}$ from the clinical database: patients' self-reported age, sex and smoking status (past, never, current); and body mass index (BMI) as measured by a sleep technician. Age and sex were confirmed with the use of health administrative data.

We used the following variables to describe our cohort at baseline: daytime sleepiness, as measured by the Epworth Sleepiness Scale score, and other polysomnographic indices (total sleep time, arousals index, total number of awakenings) were obtained from the clinical database; baseline comorbidities were identified from health administrative data over a 3-year period before the sleep study; and income status was derived from census data (details are available in Appendix 2).

\section{Statistical analysis}

To address missing values, we used multivariate imputation by chained equations to generate 5 complete datasets. ${ }^{23,24}$ To obtain a single estimate and standard error for each parameter, we pooled these results according to Rubin's rules. ${ }^{24}$

In our prevalence study, which involved the full cohort, we performed multivariable logistic regression analysis to evaluate an association between having a cancer diagnosis by the time of the sleep study (baseline) and severity of obstructive sleep apnea at baseline.

In our incidence study, which involved the sample free of cancer at baseline, we calculated crude incidence rates for cancer per 100 personyears for the entire sample and by severity of obstructive sleep apnea. ${ }^{1,17}$ In a frail population, death, termed a competing event, may preclude the occurrence of cancer and lead to overestimation of incidence by the usual Kaplan-Meier method. ${ }^{25}$ Therefore, we also estimated incidence with the cumulative incidence function, which accounts for competing risks. We tested

Table 1: Characteristics of 10149 patients at the time of a full-night diagnostic sleep study (baseline)

\begin{tabular}{|c|c|c|c|c|c|c|}
\hline \multirow[b]{2}{*}{ Characteristic } & \multicolumn{2}{|c|}{ Cancer at baseline* } & \multicolumn{4}{|c|}{ Severity of obstructive sleep apnea in sample without cancer* } \\
\hline & $\begin{array}{c}\text { Yes } \\
n=520\end{array}$ & $\begin{array}{c}\text { No } \\
n=9629\end{array}$ & $\begin{array}{l}\mathrm{AHI}<5 \\
n=2054\end{array}$ & $\begin{array}{c}\text { AHI } 5 \text { to }<15 \\
n=2600\end{array}$ & $\begin{array}{c}\text { AHI } 15 \text { to } \leq 30 \\
n=2113\end{array}$ & $\begin{array}{l}\mathrm{AHI}>30 \\
n=2793\end{array}$ \\
\hline Male, no. (\%) & $310(59.6)$ & $5974(62.0)$ & $934(45.5)$ & $1501(57.7)$ & $1358(64.3)$ & $2141(76.7)$ \\
\hline Age, yr, median (IQR) & $64(55-73.0)$ & $48(39-58)$ & $42(32-51)$ & $47(38-56)$ & $50(41-59)$ & $52(44-62)$ \\
\hline Daytime sleepiness, $†$ no. (\%) & $210(40.4)$ & $3447(35.8)$ & $677(33.0)$ & 830 (31.9) & $708(33.5)$ & $1196(42.8)$ \\
\hline \multicolumn{7}{|l|}{$\begin{array}{l}\text { Smoking status, self-reported, } \\
\text { no. }(\%)\end{array}$} \\
\hline Current & $65(12.5)$ & $1774(18.4)$ & $396(19.3)$ & $513(19.7)$ & $360(17.0)$ & $487(17.4)$ \\
\hline Former & $136(26.2)$ & $1733(18.0)$ & 275 (13.4) & $403(15.5)$ & $404(19.1)$ & $630(22.6)$ \\
\hline Never & $273(52.5)$ & $5333(55.4)$ & $1227(59.7)$ & $1456(56.0)$ & $1188(56.2)$ & $1432(51.3)$ \\
\hline \multicolumn{7}{|l|}{ Income quintile, no. (\%) } \\
\hline 1 (poorest) & $97(18.7)$ & $1893(19.7)$ & $385(18.7)$ & $464(17.8)$ & $442(20.9)$ & $589(21.1)$ \\
\hline 5 (wealthiest) & $174(33.5)$ & $2691(27.9)$ & $600(29.2)$ & $741(28.5)$ & $594(28.1)$ & $742(26.6)$ \\
\hline \multicolumn{7}{|l|}{ Polysomnography indexes } \\
\hline Total sleep time, h, median (IQR) & $5.3(4.4-6.2)$ & $5.8(4.9-6.4)$ & $5.8(5.0-6.5)$ & $5.9(5.1-6.5)$ & $5.8(5.0-6.5)$ & $5.5(4.6-6.3)$ \\
\hline AHI, total, events/h, median (IQR) & $20.8(10.4-43.1)$ & $15.5(5.9-34.4)$ & $2.1(0.9-3.5)$ & $9.3(7-11.9)$ & $21.1(17.8-25.2)$ & $49.7(38.0-70.2)$ \\
\hline $\begin{array}{l}\text { Total sleep time spent with } \\
\mathrm{SaO}_{2}<90 \% \text {, min, median (IQR) }\end{array}$ & $3.3(0.1-24.9)$ & $0.4(0.0-8.4)$ & $0(0-0.1)$ & $0.1(0-1.7)$ & $0.9(0.0-7.5)$ & $12.3(1.4-51.3)$ \\
\hline $\begin{array}{l}\text { Cumulative incidence of cancer } \\
\text { at } 5 \mathrm{yr}, \%(95 \% \mathrm{Cl})\end{array}$ & - & $3.7(3.3-4.0)$ & $2.6(1.9-3.3)$ & $2.9(2.2-3.5)$ & $4.0(3.1-4.9)$ & $4.9(4.1-5.8)$ \\
\hline
\end{tabular}


differences in incident cancer between groups using the modified log-rank statistic. ${ }^{26}$ We used multivariable Cox regression models to investigate the associations between obstructive sleep apnea and incident cancer.

In the logistic and Cox regression models, each predictor was controlled for known general risk factors for cancer development. We tested several statistical models in which the predictors (apnea-hypopnea index and sleep time spent with oxygen saturation $<90 \%$ ) were treated as both continuous and categorical variables. For continuous variables, if nonlinearity was observed, we used restricted cubic-spline transformations. A priori, we specified interactions between apnea-hypopnea index or oxygen saturation and age, sex, daytime sleepiness and BMI at baseline. . $^{3-5}$

As a secondary analysis, to replicate the results of the clinic-based study by Campos-
Rodriguez and colleagues that showed an association between obstructive sleep apnea and incident cancer, ${ }^{3}$ we used that study's categories for the apnea-hypopnea index $(<18.7,18.7-43$ and $>43$ events/h) and percent of sleep time spent with oxygen saturation below $90 \%(<1.2 \%$, $1.2 \%-12 \%$ and $>12 \%$ ). In another analysis, we refitted our statistical models for individual cancer types (as defined by anatomic location, cause and detectability by screening) that had sufficient numbers of cases.

We performed several sensitivity analyses. First, because of the presence of death as a competing risk (i.e., patients could die before being diagnosed with cancer), we modelled the causespecific hazard of incident cancer using the Fine and Gray competing-risk regression model. ${ }^{27}$ Second, in the post-2004 cohort, for whom information on claims for continuous positive airway pressure (CPAP) devices was available, we refit-

Table 2: Association between predictors related to obstructive sleep apnea and the prevalence and incidence of cancer*

\begin{tabular}{|c|c|c|}
\hline Model; predictor & $\begin{array}{c}\text { Association with cancer } \\
\text { prevalence, OR }(95 \% \mathrm{Cl}) \\
(N \text { total }=10149 \\
n \text { events }=520)\end{array}$ & $\begin{array}{c}\text { Association with cancer } \\
\text { incidence, } \mathrm{HR}(95 \% \mathrm{Cl}) \\
(N \text { total }=9629, \\
n \text { events }=627)\end{array}$ \\
\hline \multicolumn{3}{|l|}{ Univariable analysis } \\
\hline \multicolumn{3}{|l|}{$\mathrm{AHI}$ as categorical variable, events/h } \\
\hline 5 to $<15$ v. $<5$ & $1.30(0.97-1.76)$ & $1.37(1.05-1.80)$ \\
\hline 15 to $\leq 30$ v. $<5$ & $1.84(1.38-2.46)$ & $1.39(1.08-1.79)$ \\
\hline$>30$ v. $<5$ & $1.89(1.43-2.50)$ & $2.02(1.61-2.54)$ \\
\hline \multicolumn{3}{|l|}{ Multivariable analysis $†$} \\
\hline \multicolumn{3}{|l|}{ Model 1: AHI as continuous variable, events/h } \\
\hline Per 10 events & $1.02(0.98-1.06)$ & $1.01(0.98-1.04)$ \\
\hline \multicolumn{3}{|l|}{ Model 2: AHI as categorical variable, events/h } \\
\hline 5 to $<15$ v. $<5$ & $0.96(0.70-1.32)$ & $0.98(0.76-1.27)$ \\
\hline 15 to $\leq 30$ v. $<5$ & $1.14(0.83-1.55)$ & $0.96(0.76-1.23)$ \\
\hline$>30$ v. $<5$ & $0.96(0.71-1.30)$ & $1.02(0.80-1.31)$ \\
\hline Model 3: AHI as categorical variable, events/h & $1.02(0.80-1.30)$ & $1.03(0.83-1.28)$ \\
\hline \multicolumn{3}{|l|}{$>43$ v. $<18.7 \ddagger$} \\
\hline \multicolumn{3}{|l|}{ Model 4: $\mathrm{SaO}_{2}$ as continuous variable } \\
\hline Time spent with $\mathrm{SaO}_{2}<90 \%$, min, per $10 \mathrm{~min}$ & $1.01(1.00-1.03) \S$ & $1.00(0.99-1.02)$ \\
\hline \multicolumn{3}{|l|}{ Model 5: $\mathrm{SaO}_{2}$ as categorical variable } \\
\hline $\begin{array}{l}\% \text { of sleep time spent with } \mathrm{SaO}_{2}<90 \% \text {, } \\
>12 \% \text { v. }<1.2 \% \ddagger\end{array}$ & $1.31(1.00-1.71) \uparrow$ & $1.13(0.89-1.42)$ \\
\hline \multicolumn{3}{|c|}{$\begin{array}{l}\text { Note: } \mathrm{AHI}=\text { apnea-hypopnea index, } \mathrm{Cl}=\text { confidence interval, } \mathrm{HR}=\text { hazard ratio, } \mathrm{OR}=\text { odds ratio, } \mathrm{SaO}_{2}=\text { oxygen saturation. } \\
{ }^{*} \text { To obtain a single estimate and standard error for each parameter, the results from the } 5 \text { imputed datasets were pooled } \\
\text { according to Rubin's rules. }{ }^{24} \\
\text { tModels were adjusted for age, sex, body mass index and smoking status. } \\
\text { tTo attempt to replicate the results of only one clinic-based study that evaluated an association between obstructive sleep } \\
\text { apnea and incident cancer, }{ }^{3} \text { we used that study's categories for } \mathrm{AHI}(<18.7,18.7-43 \text { and }>43 \text { events/h) and for percent of sleep } \\
\text { time spent with } \mathrm{SaO}_{2}<90 \%(<1.2 \%, 1.2 \%-12 \% \text { and }>12 \%) \text {. } \\
\$ p=0.09 \text { for dataset } 1, p=0.04 \text { for dataset } 2, p=0.2 \text { for dataset } 3, p=0.04 \text { for dataset } 4, p=0.09 \text { for dataset } 5 \text {. } \\
\text { T } p=0.049 \text { for dataset } 1, p=0.06 \text { for dataset } 2, p=0.09 \text { for dataset } 3, p=0.02 \text { for dataset } 4, p=0.045 \text { for dataset } 5 \text {. }\end{array}$} \\
\hline
\end{tabular}


ted statistical models with additional censoring at the time of CPAP claim to include only patients who had not claimed CPAP devices. Additional sensitivity analyses included adjustment for the possibility that lag time would exist between the diagnosis of obstructive sleep apnea and the diagnosis of cancer, and adjustment for the number of primary care visits within the year before the diagnostic sleep study.

All statistical analyses were performed using $\mathrm{R}$ version 2.15.2 (www.r-project.org).

\section{Results}

In total, 10149 patients were included in the full cohort. The proportion of patients missing data was $0.69 \%$ for the apnea-hypopnea index and $10.1 \%$ for sleep time spent with oxygen saturation below 90\%. Details about this cohort are reported elsewhere. ${ }^{11}$

\section{Prevalent cancer and obstructive sleep apnea}

A total of 520 patients $(5.1 \%)$ had prevalent cancer at baseline. They were older, were more likely to be former smokers, had a greater number of comorbid conditions and had more severe obstructive sleep apnea than the patients without a cancer diagnosis at baseline (Table 1). In the unadjusted logistic regression, severity of obstructive sleep apnea was significantly associated with prevalent cancer (odds ratio [OR] for apnea-hypopnea index > 30 v. < 5: 1.89, 95\% confidence interval [CI] 1.43-2.50; OR per 10 -minute increase in sleep time spent with oxygen saturation < 90\%: 1.04 , 95\% CI 1.02-1.05). The effect became nonsignificant after we controlled for age, sex, BMI and smoking status (Table 2).

\section{Incident cancer and obstructive sleep apnea}

Over a median follow-up of 93 months, 627 $(6.5 \%)$ of the 9629 patients who were free of cancer at the time of the sleep study received a diagnosis of incident cancer, for an incidence rate of 0.82 per 100 person-years. Prostate $(n=$ $125)$, breast $(n=75)$, colorectal $(n=64)$ and lung $(n=61)$ cancers were the most common. The potential competing event, death without diagnosed cancer, occurred in 630 patients (total number of deaths $=858$ ). The cumulative incidence of cancer at 5 years was $3.7 \%(95 \% \mathrm{CI}$ $3.3 \%-4.0 \%$ ) overall, and ranged from $2.6 \%$ (apnea-hypopnea index $<5$ ) to $4.9 \%$ (apneahypopnea index > 30) (Table 1, Figure 2). The unadjusted difference in incidence of cancer was significant between patients who had severe obstructive sleep apnea and others $(p<0.001)$, but not between patients who had moderate and mild obstructive sleep apnea and those whose apnea-hypopnea index was less than $5(p>0.3)$.

In the Cox regression analyses, the crude significant effect of severity of obstructive sleep apnea at baseline on incident cancer became nonsignificant after we controlled for age, sex, BMI and smoking status (Table 2). The association remained nonsignificant when we applied the categories for predictors of obstructive sleep apnea used by Campos-Rodriguez and colleagues. ${ }^{3}$ Age, sex, BMI or daytime sleepiness did not change the strength of the association between obstructive sleep apnea-related predictors and incident cancer: interactions tested were not significant $(p>0.2)$. The results did not change when we considered death as a competing risk in the Fine and Gray regression. Finally, the same negative results were obtained when the models were restricted to patients who did not claim CPAP treatment. Other factors related to obstructive sleep apnea (daytime sleepiness, total sleep time, arousals index and total number of awakenings) that tested as possible risk factors were not significantly associated with an increased risk of all-cause mortality.

The sensitivity analyses in which we adjusted for the possibility that 1 or 2 years of lag time would exist between the diagnosis of obstructive sleep apnea and a diagnosis of cancer, and for

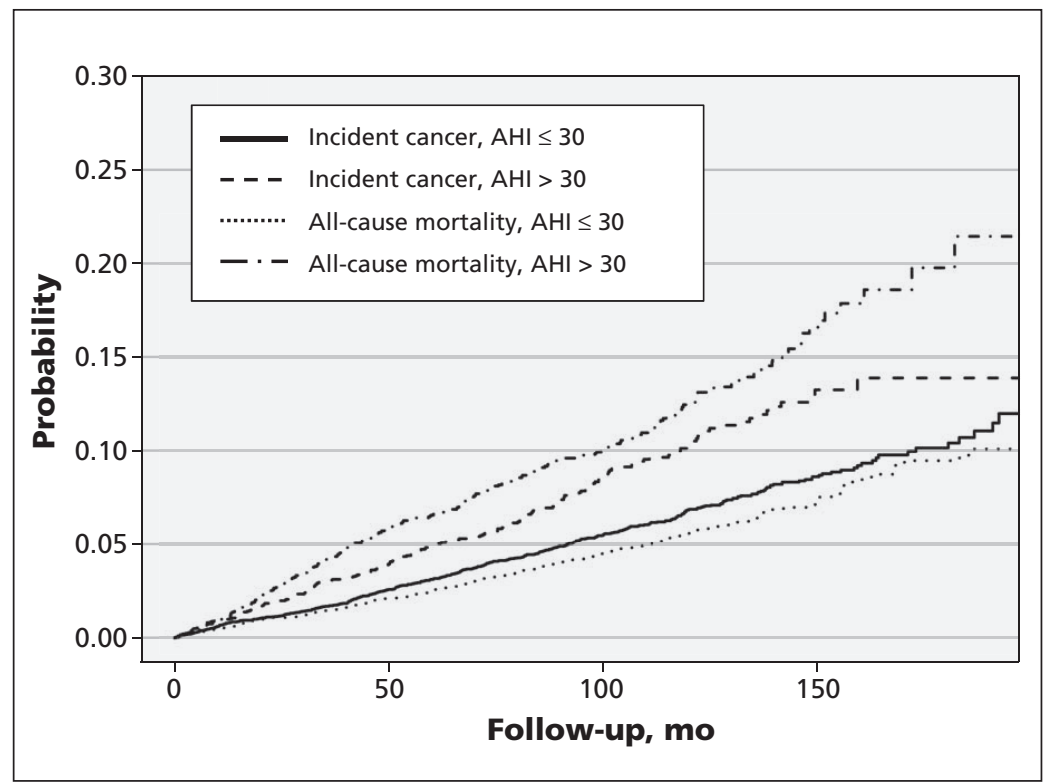

Figure 2: Estimated cumulative incidence of incident cancer and all-cause mortality, by severity of obstructive sleep apnea (as measured by apnea-hypopnea index $[\mathrm{AHI}])$. At 60 months, the cumulative incidence of cancer was $4.9 \%(95 \%$ confidence interval $[\mathrm{Cl}] 4.1 \%-5.8 \%$ ) among patients with an $\mathrm{AHI}$ greater than 30 and $3.1 \%(95 \% \mathrm{Cl} 2.7 \%-3.6 \%)$ among those with a lower index. The corresponding rates at 120 months were $10.2 \%$ (95\% Cl $8.7 \%-11.7 \%)$ among patients with an $\mathrm{AHI}$ greater than 30 and $6.9 \%$ (95\% Cl 6.2\%-7.6\%) among those with a lower index. 
the number of primary care visits within the year before the diagnostic sleep study, gave results similar to those of the main analyses (Appendix 3, available at www.cmaj.ca/lookup/suppl/doi :10.1503/cmaj.140238/-/DC1).

When we evaluated the association between severity of obstructive sleep apnea and different types of cancer, we found a significant association between oxygen desaturation and smokingrelated cancers (Appendix 3).

\section{Interpretation}

In our population study using clinical and health administrative data from a large cohort of more than 10000 patients who had suspected obstructive sleep apnea, we found that severity of obstructive sleep apnea - expressed by apneahypopnea index or oxygen desaturation during sleep time - was not associated with overall prevalent or incident cancer after we controlled for known cancer-related risk factors. Thus, we were not able to confirm previous hypotheses that obstructive sleep apnea is a cause of overall cancer development through intermittent hypoxemia. ${ }^{28}$ However, in subgroup analyses, we found that the level of oxygen desaturation was associated with the development of smokingrelated cancers.

The results of our study are consistent with those from a large community-based study that found no association between incident cancer and obstructive sleep apnea defined by symptoms. ${ }^{4}$

Our results are not consistent with the clinicbased study by Campos-Rodriguez and colleagues. ${ }^{3}$ In their study, oxygen desaturation, but not the apnea-hypopnea index, was found to be associated with incident cancer in a fully adjusted model (hazard ratio [HR] for percent of sleep time with oxygen saturation below $90 \%$ $>12 \%$ v. $<1.2 \%$ : $2.33,95 \%$ CI $1.57-3.46$; HR for apnea-hypopnea index $>43$ v. $<18.7$ events/h: $1.17,95 \%$ CI $0.84-1.65){ }^{3}$ Similar to our study, the authors found colorectal, prostate, lung and breast cancers to be the most common, and the cumulative incidence of cancer at 5 years was about $5 \%$ among people with severe obstructive sleep apnea. However, when we applied the same categories for predictors of obstructive sleep apnea used in their study, ${ }^{3,24}$ we were unable to replicate their findings (Table 2). There are several reasons why our results might have differed. First, differences in study participants: their cohort appeared to be older, with higher BMI and more severe obstructive sleep apnea, and therefore it could have had more patients with oxygen desaturation, which was more likely to result in cancer development. Our study had a longer follow-up time (median $7.8 \mathrm{yr}$ v. $4.5 \mathrm{yr}$ ) and a larger number of incident cancers (627 v. 261), which we believe made it more likely to detect the "true" effect of obstructive sleep apnea on cancer development. Another reason our results may have differed was that adherence to CPAP and other treatments of obstructive sleep apnea were unaccounted for in our study and may have attenuated the effects of obstructive sleep apnea-related predictors. Our results, however, remained unchanged when we restricted the analysis to patients who did not claim CPAP treatment. Finally, we controlled for the competing risk of death and used data from consistently collected full polysomnographic recordings to minimize measurement bias.

Our results also do not agree with those from the studies by Nieto and colleagues ${ }^{5}$ and Marshall and colleagues, ${ }^{8}$ which found that people who had moderate to severe obstructive sleep apnea were more likely than those without the condition to have higher cancer incidence and mortality (Appendix 1). However, the relatively small number of events and patients with severe obstructive sleep apnea led to wide CIs, which indicated a high level of uncertainty around this association (Appendix 1). Their results may also be explained by a significant association between severity of obstructive sleep apnea and all-cause mortality, ${ }^{11,29-31}$ and a bias toward classifying someone as dying of cancer if they have cancer, when in fact they may have died of another cause or their cancer may have been only a secondary cause of death. ${ }^{32}$

In our subgroup analyses, similar to the study by Campos-Rodriguez and colleagues, ${ }^{3}$ we found that oxygen desaturation, but not the apneahypopnea index, was significantly associated with smoking-related cancers. Hypoxia has been shown to cause upregulation and overexpression of a number of genes related to biological behaviours of cancer cells, including apoptosis, metastasis and angiogenesis, ${ }^{33}$ which renders the cancer cells more aggressive and resistant to therapy. ${ }^{34}$ Indeed, chronic intermittent hypoxia has been shown to accelerate cancer progression in a mouse model of sleep apnea. ${ }^{6,35}$ It is conceivable that the carcinogenic effects of some tobacco compounds may be exacerbated by intermittent hypoxemia or the inflamed upper-airway environment associated with obstructive sleep apnea. ${ }^{36,37}$ However, the results from our subgroup analyses need to be interpreted with caution.

\section{Strengths and limitations}

Our study had many strengths, including long and complete follow-up, a relatively large num- 
ber of events, consistently collected polysomnographic data using the same polysomnographic scoring criteria over time, a wide range of obstructive sleep apnea severity, and access to comprehensive validated cancer registry.

The main limitation of our study is its observational, historical, single-centre design, which limits its generalizability and increases the potential for unmeasured confounding. For example, we were unable to retrieve information about cancer stage and management, and we assumed that patients with cancer were managed equally according to the Ontario standards. These factors could have led to unmeasured confounding. However, we controlled for a number of known risk factors for cancer development. Also, we examined a mixture of types of cancer with various causes, pathophysiology and risk factors; a strong association of one type with obstructive sleep apnea (e.g., certain types are more susceptible to low oxygen concentrations) might have been attenuated by a weaker association of another. Finally, we had insufficient power to examine common cancer subtypes because of the small number of events.

\section{Conclusion}

In our study of a large cohort with varying degrees of obstructive sleep apnea, severity of obstructive sleep apnea was not found to be independently associated with either prevalent or incident cancer, except in one subgroup analysis of smoking-related cancers. Additional studies are needed to elucidate whether there is an independent association between obstructive sleep apnea and specific types of cancer.

\section{References}

1. Epstein LJ, Kristo D, Strollo PJ Jr, et al. Clinical guideline for the evaluation, management and long-term care of obstructive sleep apnea in adults. J Clin Sleep Med 2009;5:263-76.

2. Evans J RC, McRae L, Driver H, Fleetham J. The prevalence rate and risk of obstructive sleep apnea in Canada. Chest 2010; 138(4_MeetingAbstracts):702A-A.

3. Campos-Rodriguez F, Martinez-Garcia MA, Martinez M, et al. Association between obstructive sleep apnea and cancer incidence in a large multicenter Spanish cohort. Am J Respir Crit Care Med 2013; 187:99-105.

4. Christensen AS, Clark A, Salo P, et al. Symptoms of sleep disordered breathing and risk of cancer: a prospective cohort study. Sleep 2013;36:1429-35.

5. Nieto FJ, Peppard PE, Young T, et al. Sleep-disordered breathing and cancer mortality: results from the Wisconsin Sleep Cohort Study. Am J Respir Crit Care Med 2012;186: 190-4.

6. Almendros I, Montserrat JM, Torres M, et al. Intermittent hypoxia increases melanoma metastasis to the lung in a mouse model of sleep apnea. Respir Physiol Neurobiol 2013;186: 303-7.

7. Redline S, Quan SF. Sleep apnea: A common mechanism for the deadly triad - cardiovascular disease, diabetes, and cancer? Am J Respir Crit Care Med 2012;186:123-4.

8. Marshall NS, Wong KK, Cullen SR, et al. Sleep apnea and 20-year follow-up for all-cause mortality, stroke, and cancer incidence and mortality in the busselton health study cohort. J Clin Sleep Med 2014;10:355-62.
9. Peppard PE, Nieto FJ. Here come the sleep apnea-cancer studies. Sleep 2013;36:1409-11.

10. Kim R, Kapur VK. Emerging from the shadows: a possible link between sleep apnea and cancer. J Clin Sleep Med 2014;10: 363-4.

11. Kendzerska T, Gershon AS, Hawker G, et al. Obstructive sleep apnea and risk of cardiovascular events and all-cause mortality: a decade-long historical cohort study. PLoS Med 2014;11: e1001599.

12. Robles SC, Marrett LD, Clarke EA, et al. An application of capturerecapture methods to the estimation of completeness of cancer registration. J Clin Epidemiol 1988;41:495-501.

13. McLaughlin JR, Kreiger N, Marrett LD, et al. Cancer incidence registration and trends in Ontario. Eur J Cancer 1991;27: 1520-4.

14. Hall S, Schulze K, Groome P, et al. Using cancer registry data for survival studies: the example of the Ontario Cancer Registry. J Clin Epidemiol 2006;59:67-76.

15. Brenner DR, Tammemagi MC, Bull SB, et al. Using cancer registry data: agreement in cause-of-death data between the Ontario Cancer Registry and a longitudinal study of breast cancer patients. Chronic Dis Can 2009;30:16-9.

16. Sleep-related breathing disorders in adults: recommendations for syndrome definition and measurement techniques in clinical research. The Report of an American Academy of Sleep Medicine Task Force. Sleep 1999;22:667-89.

17. Fleetham J, Ayas N, Bradley D, et al. Canadian Thoracic Society guidelines: diagnosis and treatment of sleep disordered breathing in adults. Can Respir J 2006;13(7):387-92.

18. North CM, Christiani DC. Women and lung cancer: What is new? Semin Thorac Cardiovasc Surg 2013;25:87-94.

19. Parkin DM. 1. The fraction of cancer attributable to lifestyle and environmental factors in the UK in 2010. Br J Cancer 2011;105 (Suppl 2):S2-5

20. Parkin DM, Boyd L, Walker LC. 16. The fraction of cancer attributable to lifestyle and environmental factors in the UK in 2010. Br J Cancer 2011;105(Suppl 2):S77-81.

21. Stephenson GD, Rose DP. Breast cancer and obesity: an update. Nutr Cancer 2003;45:1-16.

22. Calle EE, Rodriguez C, Walker-Thurmond K, et al. Overweight, obesity, and mortality from cancer in a prospectively studied cohort of US adults. N Engl J Med 2003;348:1625-38.

23. Azur MJ, Stuart EA, Frangakis C, et al. Multiple imputation by chained equations: What is it and how does it work? Int J Methods Psychiatr Res 2011;20:40-9.

24. Rubin DB. Multiple imputation for nonresponse in surveys. New York: Wiley; 1987.

25. Wolbers M, Koller MT, Witteman JC, et al. Prognostic models with competing risks: methods and application to coronary risk prediction. Epidemiology 2009;20:555-61.

26. Gray RJ. A class of $K$-sample tests for comparing the cumulative incidence of a competing risk. Ann Stat 1988;16:1141-54.

27. Fine JP, Gray RJ. A proportional hazards model for the subdistribution of a competing risk. J Am Stat Assoc 1999;94: 496-509.

28. Abrams B. Cancer and sleep apnea - the hypoxia connection. Med Hypotheses 2007;68:232.

29. Punjabi NM, Caffo BS, Goodwin JL, et al. Sleep-disordered breathing and mortality: a prospective cohort study. PLoS Med 2009; 6:e1000132.

30. Young T, Finn L, Peppard PE, et al. Sleep disordered breathing and mortality: eighteen-year follow-up of the Wisconsin sleep cohort. Sleep 2008;31:1071-8.

31. Lavie P, Lavie L, Herer P. All-cause mortality in males with sleep apnoea syndrome: declining mortality rates with age. Eur Respir J 2005;25:514-20.

32. Black WC, Haggstrom DA, Welch HG. All-cause mortality in randomized trials of cancer screening. J Natl Cancer Inst 2002;94: 167-73.

33. Liu Y, Song X, Wang X, et al. Effect of chronic intermittent hypoxia on biological behavior and hypoxia-associated gene expression in lung cancer cells. J Cell Biochem 2010;111:554-63.

34. Wouters A, Pauwels B, Lardon F, et al. Review: implications of in vitro research on the effect of radiotherapy and chemotherapy under hypoxic conditions. Oncologist 2007;12:690-712.

35. Almendros I, Montserrat JM, Torres M, et al. Obesity and intermittent hypoxia increase tumor growth in a mouse model of sleep apnea. Sleep Med 2012;13:1254-60.

36. Hoffmann D, Hecht SS. Advances in tobacco carcinogenesis. In: Cooper CS, Grover PL, editors. Handbook of experimental pharmacology. Vol 94. Berlin: Springer-Verlag; 1990:63-102.

37. Boyd JH, Petrof BJ, Hamid Q, et al. Upper airway muscle inflammation and denervation changes in obstructive sleep apnea. Am J Respir Crit Care Med 2004;170:541-6. 
Affiliations: Institute of Health Policy, Management and Evaluation (Tomlinson), University of Toronto; Institute for Clinical Evaluative Sciences (Kendzerska, Hawker, Gershon); Women's College Research Institute (Kendzerska, Hawker), Women's College Hospital, University of Toronto; Department of Medicine (Kendzerska, Leung, Hawker, Gershon), University of Toronto; Department of Medicine (Leung), St. Michael's Hospital; Department of Medicine (Tomlinson), University Health Network and Mount Sinai Hospital; Department of Medicine (Gershon), Sunnybrook Health Sciences Centre, Toronto, Ont.

Contributors: Tetyana Kendzerska was involved in the study concept and design, the collection of administrative data, and the analysis and interpretation of data. Richard Leung, Gillian Hawker, George Tomlinson and Andrea Gershon were involved in the study design and interpretation of data. Richard Leung also was involved in the study concept and is holder of the sleep portion of the chest dataset from which the study sample was extracted. George Tomlinson also was involved in the analysis of data and the drafting of the methods and results sections of the manuscript. Andrea Gershon also was involved in the study concept, the collection of administrative data and the anal- ysis of data. Tetyana Kendzerska drafted the manuscript, and the other authors critically revised it. All of the authors gave final approval of the version to be published and agreed to act as guarantors of the work.

Funding: The study was supported by the ResMed Foundation and by the Institute for Clinical Evaluative Sciences (ICES). ICES is funded by an annual grant from the Ontario Ministry of Health and Long-Term Care. The opinions, results and conclusions reported in this paper are those of the authors and are independent from the funding sources. No endorsement by ICES, the Ontario Ministry of Health and Long-Term Care or the ResMed Foundation is intended or should be inferred.

Tetyana Kendzerska is supported by a Canadian Institutes of Health Research (CIHR) doctoral research award. Gillian Hawker is supported by the F.M. Hill Chair in Academic Women's Medicine. Andrea Gershon is supported by the Physicians' Services Incorporated Foundation Fellowship in Translational Health Research.

Acknowledgement: The authors thank Dr. Victor Hoffstein for creating and maintaining the St. Michael's Hospital Sleep Laboratory database.

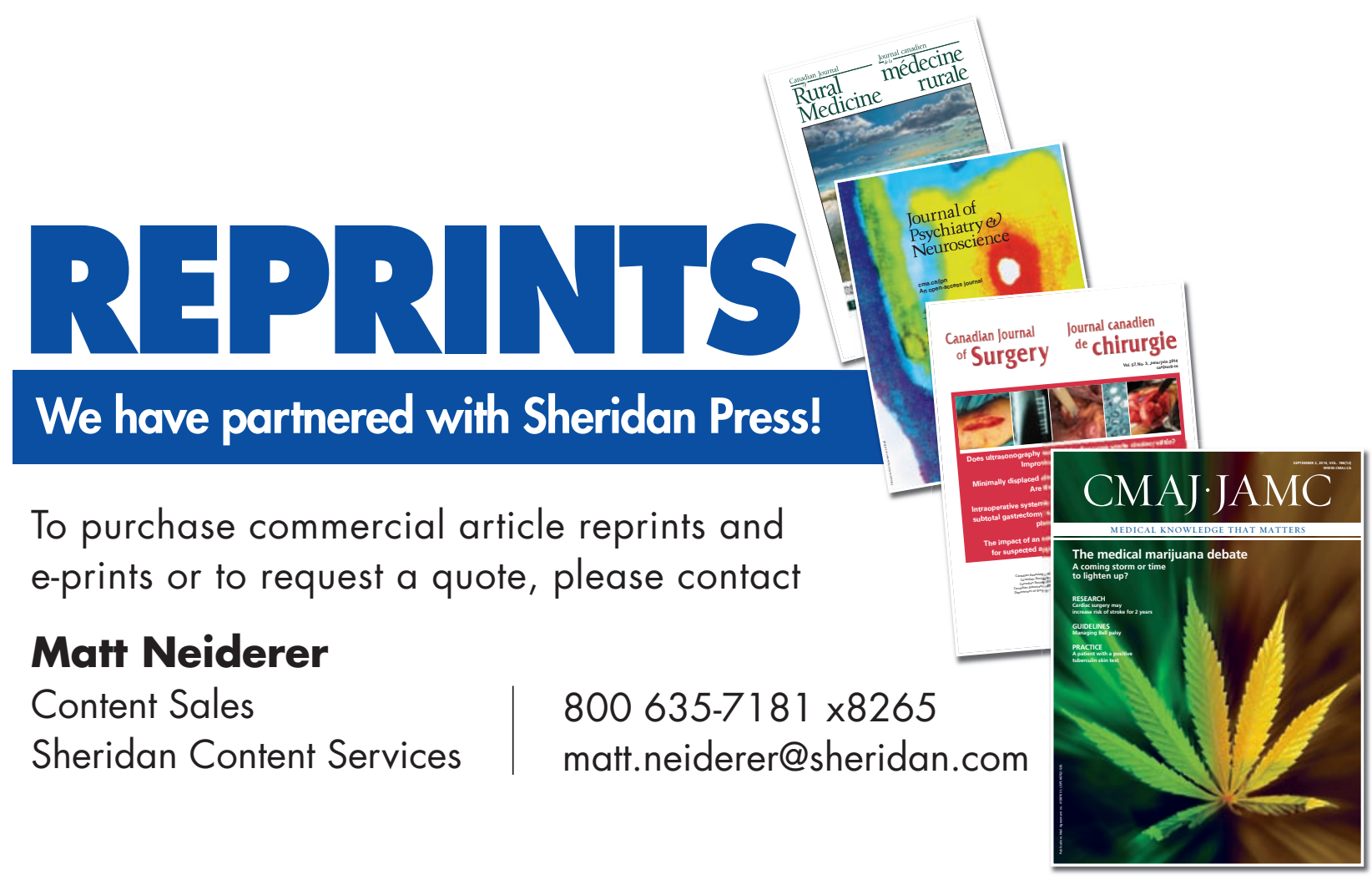

